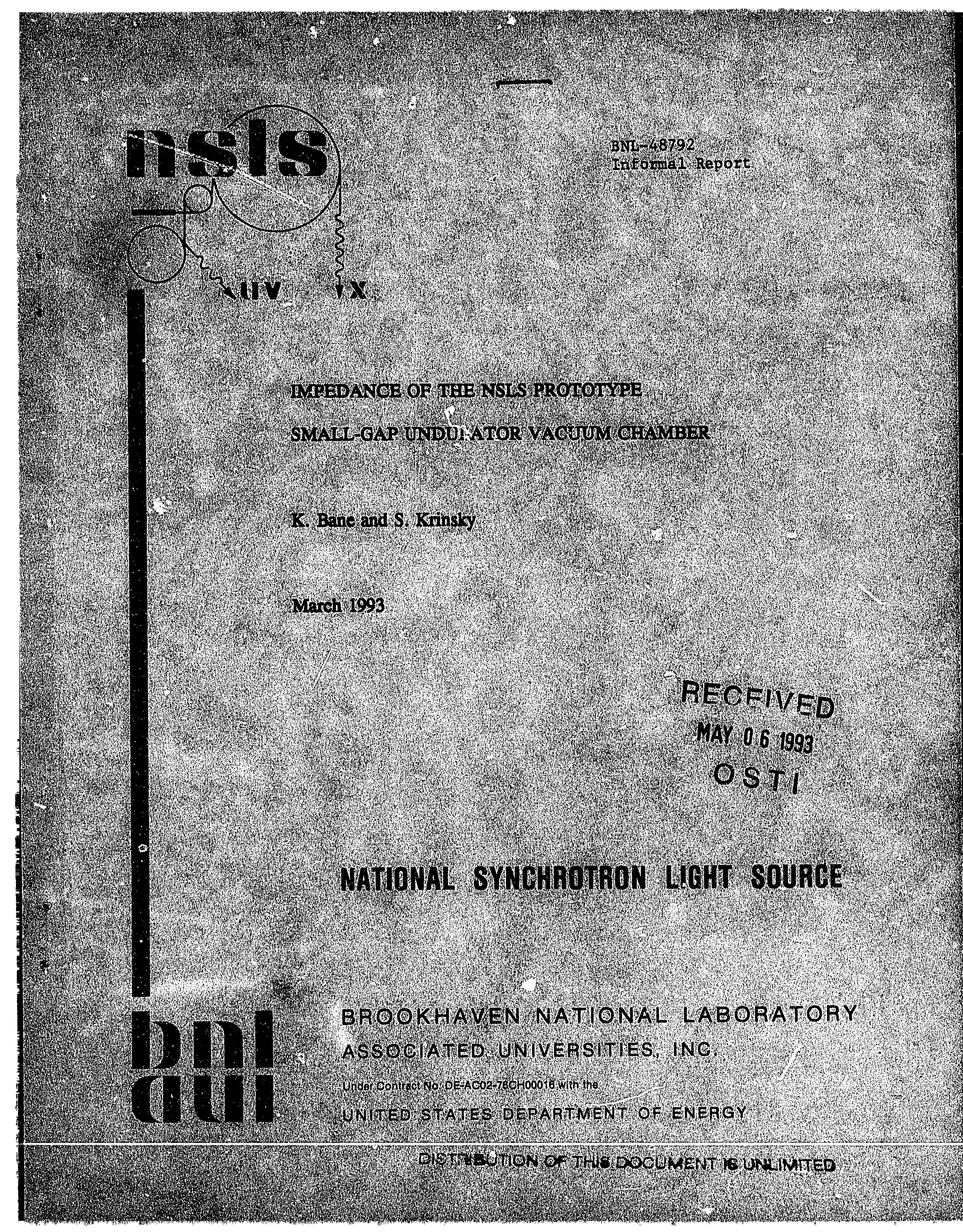




\title{
IMPEDANCE OF THE NSLS PROTOTYPE \\ SMALL-GAP UNDULATOR VACUUM CHAMBER*
}

\author{
K. Bane \\ Stanford Linear Accelerator Center \\ Stanford, CA 94309 \\ S. Krinsky \\ National Synchrotron Light Source \\ Brookhaven National Laboratory \\ Upton, NY 11973
}

\begin{abstract}
Insertion device performance is limited by the minimum magnet gap allowed by storage ring beam dynamics. In this note, we analyze the impedance of the vacuum chamber for the prototype small-gap undulator being built for the NSLS X-Ray ring, and discuss the consequent beam instability thresholds.
\end{abstract}

"Work performed under the auspices of the U.S. Department of Energy under contract No. DE-AC02-76CH00016. 


\section{INTRODUCTION}

In order to optimize insertion device performance, we wish to operate with the minimum magnet gap allowed by the electron beam dynamics. A prototype small gap undulator ${ }^{1}$ (PSGU) is sunder" construction for use in the X13 straight section of the X-Ray ring. In this note we consider the impedance of the PSGU variable aperture vacuum chamber and discuss its effect on beam stability.

The PSGU chamber is made of $1 \mathrm{~mm}$ thick stainless steel, and is approximately $30 \mathrm{~cm}$ in length. It has an inner aperture which can be adjusted from $20 \mathrm{~mm}$ down to $2 \mathrm{~mm}$ (full). The device is situated at the center of a low- $\beta$ insertion having values of the betatron functions $\beta_{\mathrm{x}}{ }^{*}=1.5 \mathrm{~m}$ and $\beta_{\mathrm{y}}{ }^{*}=0.33 \mathrm{~m}$ at the insertion center. The small value of the vertical betatron function significantly reduces the effect of the chamber's transverse impedance on the stability of the vertical betatron oscillations. Our results indicate that beam stability should be maintained with a $4 \mathrm{~mm}$ inner vertical aperture.

In Section II, we consider the resistive wall impedance, estimating the power dissipation in the chamber wall, the growth rate of the transverse coupled bunch instability due to the longrange part of the wakefield, and the threshold of the strong head-tail instability due to the shortrange resistive wall wakefield. In Section III, we discuss the transverse geometric wakefield due to the aperture change at the PSGU chamber transitions. In Section IV, TBCI is used to determine the longitudinal geometric wakefield, and to estimate the inductance due to the chamber transitions.

\section{KESISTIVE WALL IMPEDANCE}

In the small gap undulator, the vacuum chamber is comprised of $1 \mathrm{~mm}$ thick stainless steel, with resistivity $\rho=80 \times 10^{-8} \Omega-\mathrm{m}$. The circumference of the X-Ray ring is $170 \mathrm{~m}$, so the effective radius $R=27 \mathrm{~m}$. At the angular revolution frequency $\omega_{0}=c / R=11 \mathrm{MHz}$, the skin depth in the chamber is ${ }^{2}$

$$
\delta_{0}=\sqrt{\frac{2 p}{\mu_{0} \omega_{0}}}=0.34 \mathrm{~mm},
$$

and the surface impedance is

$$
Z_{s}=(1-i) R_{0}
$$

where

$$
x_{0}=\rho / \delta_{0}=2.4 \times 10^{-3} \mathrm{Q} .
$$


The longitudinal resistive wall impedance at the nth harmonic of the revolution frequency, $\omega=$ $n \omega_{0}$, is $^{3}$

$$
\begin{aligned}
& Z_{n}=\left(\frac{\ell}{2 \pi a}\right) Z_{s} \sqrt{n}, \quad(n \geq 0) \\
& Z_{-n}=Z_{n}^{*}
\end{aligned}
$$

where $\ell$ is the length of the small gap chamber and a is the distance of the electron beam to the chamber wall.

\section{A. Power Dissipation} chamber is

In the case of one bunch with DC current $I_{0}$ (Amperes), the power dissipation in the

$$
P=I_{0}^{2} \sum_{n=-\infty}^{\infty}\left(\operatorname{Re} Z_{n}\right) e^{-n^{2} \omega_{0}^{2} \sigma_{r}^{2}}
$$

where $\sigma_{\mathrm{t}}=\sigma_{\mathrm{s}} / \mathrm{c}$ is the one standard deviation bunch length divided by the speed of light. Approximating the sum by an integral, one obtains ${ }^{4}$

$$
P \approx I_{0}^{2}\left(\operatorname{Re} Z_{1}\right) \frac{\Gamma(3 / 4)}{\left(\omega_{0} \sigma_{0}\right)^{3 / 2}},
$$

where the gamma function $\Gamma(3 / 4)=1.2$ can be approximated by unity. When an average current $I_{a v}$ is distributed uniformly in $M$ bunches, the power dissipation is

$$
P \approx M\left(\frac{I_{a v}}{M}\right)^{2}\left(\frac{\ell}{2 \pi a}\right) \Re_{0}\left(\frac{R}{\sigma_{s}}\right)^{3 / 2},
$$

We now consider the parameters

$$
\begin{array}{ll}
\text { chamber length } & \ell=0.3 \mathrm{~m} \\
\text { effective radius } & R=27 \mathrm{~m} \\
\text { bunch length } & \sigma_{8}=0.05 \mathrm{~m}
\end{array}
$$

From Eq. (2.4) we find the power dissipation

$$
P=\frac{1400 I_{a v}^{2}(a m p)}{M a(\mathrm{~mm})}
$$


In the case of $\mathrm{a}=1 \mathrm{~mm}$, we find

\begin{tabular}{lcl}
$\mathrm{I}_{\mathrm{av}}$ & $\mathrm{M}$ & $\mathrm{P}$ \\
\cline { 3 - 3 } $0.25 \mathrm{Amp}$ & 25 & 3.5 watt \\
0.25 & 5 & 17.5 \\
0.10 & 1 & 14.0
\end{tabular}

B. Transverse Coupled Bunch Instability

by $^{3}$

The transverse resistive wall impedance $Z_{\perp}$ is related to the longitudinal impedance $Z_{\|}$

$$
Z_{\perp}=\frac{2 R}{a^{2}} \frac{Z_{\|}}{n}
$$

hence

$$
Z_{\perp}=\frac{2 R}{a^{2}} \frac{\ell}{2 \pi a} Z_{s} \frac{1}{\sqrt{n}}
$$

The transverse impedance can give rise to a transverse coupled bunch instability. The growth rate of the fastest growing coupled bunch mode is 5

$$
\frac{1}{\tau_{g r}} \approx \frac{e c}{4 \pi E} \frac{\beta^{*}}{R} I_{\alpha v} \operatorname{ReZ}_{\perp}(n=1) \frac{1}{\sqrt{1-q}},
$$

where $\beta^{*}$ is the average value of the vertical betatron function in the small gap chamber, which in our case is approximately given by the minimum value in the insertion $\left(\beta^{*} \approx 0.33 \mathrm{~m}\right)$. E $=2.5 \mathrm{GeV}$ is the electron energy and $q=0.2$ is the fractional part of the vertical betation tune $\nu_{\mathrm{y}}=6.2$. Taking $\ell=0.3 \mathrm{~m}, \mathrm{R}=27 \mathrm{~m}$, we find from Eq. (2.7)

$$
R e Z_{\perp}=\frac{6 \times 10^{6}}{[a(\mathrm{~mm})]^{3}} \Omega / \mathrm{m},
$$

and from Eq. (2.8)

$$
\frac{1}{\tau_{g r}}=\left(720 \sec ^{-1}\right) \frac{I_{a v}}{[a(m m)]^{3}} .
$$

The radiation damping time is $5 \mathrm{msec}$ at $2.5 \mathrm{GeV}$, hence radiation damping is sufficient to provide stability at $0.25 \mathrm{Amp}$. In fact, the head-tail damping arising from running with positive chromaticity is stronger than radiation damping, so transverse instability should not be a problem due to coupled bunch effects even for $a=1 \mathrm{~mm}$. 


\section{Strong Head-Tail Instability}

The short range part of the transverse resistive wall wakefield can give rise to the strong head-tail instability, a single bunch effect. The wakefield $\mathrm{W}_{\perp 0}(\mathrm{~s})$ due to a point bunch is related to the transverse impedance by ${ }^{6}$

$$
Z_{\perp}(\omega)=\frac{-i}{c} \int_{0}^{\infty} W_{\perp 0}(s) e^{i \omega s / c} d s,
$$

and for the resistive wall impedance of Eq. (2.7)

$$
W_{\perp 0}(s)=\left\{\begin{array}{cc}
\frac{2}{\pi a^{3}} \sqrt{\frac{c \rho}{4 \pi e_{0}}} \frac{1}{\sqrt{s}} & (s>0) \\
0 & (s<0)
\end{array}\right.
$$

The wakefield resulting from a Gaussian bunch is ${ }^{6}$

$$
\begin{aligned}
W_{\perp}\left(s, \sigma_{s}\right) & =\int_{0}^{\infty} d s^{\prime} \frac{1}{\sqrt{2 \pi} \sigma_{s}} e^{-(s-s)^{2} / 2 \sigma_{s}^{2}} W_{\perp 0}\left(s^{\prime}\right) \\
= & \frac{2}{\pi a^{3}} \sqrt{\frac{c \rho}{4 \pi e_{0}}} \sqrt{\frac{2}{\pi \sigma_{s}}} g\left(s / \sigma_{s}\right)
\end{aligned}
$$

where

$$
g(\xi)=e^{-\xi^{2} / 2} \int_{0}^{\infty} e^{\xi x^{2}} e^{-x^{4} / 2} d x
$$

The function $g(\xi)$ is plotted in Fig. 1 , for $-3 \leq \xi \leq 3$. The maximum value of $g(\xi)$ is g $=1.28$.

Let us define the tune shift

$$
\Delta v=\frac{e Q W_{\perp} \beta^{*} \ell}{4 \pi E}
$$


where $\hat{W}_{\perp}$ is the maximum value of the wakefield,

$$
\hat{W}_{\perp}=\frac{2}{\pi a^{3}} \sqrt{\frac{c \rho}{4 \pi e_{0}}} \sqrt{\frac{2}{\pi \sigma_{s}}}(1.28),
$$

and $\mathrm{Q}=\mathrm{Ne}$ is the total charge of the electron bunch. The threshold of the strong head tail instability is expected to occur when ${ }^{7}$

$$
\Delta v \approx v_{s},
$$

where $\nu_{\mathrm{s}}$ is the synchrotron oscillation tune, which in the X-Ray ring has a value $\nu_{\mathrm{s}}=2 \times 10^{-3}$.

The maximum value $\hat{\mathrm{W}}_{\perp}$ of the transverse wakefield is found from Eq. (2.17) to be

$$
\hat{W}_{\perp}=\frac{4.7 \times 10^{15}}{[a(m m)]^{3}} \Omega / m^{2} s,
$$

for $\sigma_{\mathrm{s}}=0.05 \mathrm{~m}$. An average current of $1 \mathrm{Amp}$ in a single bunch corresponds to $3 \times 10^{12}$ electrons, or a total charge $\mathrm{Q}=480 \mathrm{nC}$. Taking $\ell=0.3 \mathrm{~m}, \beta^{*}=0.33 \mathrm{~m}, \mathrm{E}=2.5 \mathrm{GeV}$, the tune shift defined in Eq. (2.16) is

$$
\Delta v=8 \times 10^{-3} \frac{I_{0}(\mathrm{Amp})}{[a(\mathrm{~mm})]^{3}}
$$

For $100 \mathrm{ma}$ in a single bunch and a $=1 \mathrm{~mm}$, we find $\Delta \nu=0.8 \times 10^{-3}$, which is slightly less than the synchrotron tune $\nu_{\mathrm{s}}=2 \times 10^{-3}$. We should be close to threshold for the strong head tail instability in this case. However, taking a $=2 \mathrm{~mm}$, the tune shift is reduced to $\Delta \nu=10^{-4}$, well below threshold. If in addition, we consider 250 ma uniformly distributed in 25 bunches, then $\mathrm{I}_{\mathrm{o}}=10 \mathrm{ma}$, and the tune shift $\Delta \nu=10^{-5}$. Therefore, in this operating case, we are very far from the threshold of the strong head tail instability.

\section{Vertical Emittance Blowup}

Another concern is that the transverse wakefield might increase the verticai emittance. Electrons at the front of the bunch receive no vertical kick, but electrons in the bunch center or rear will be kicked. The vertical emittance of the X-Ray ring is $10^{-9} \mathrm{~m}$-rad, and $\beta^{*}=0.33 \mathrm{~m}$, hence $\sigma_{\mathrm{y}}{ }^{\prime}=5 \times 10^{-5} \mathrm{rad}$ in the insertion. 
is $^{7}$

The maximum angular kick experienced by an electron due to the transverse wakefield

$$
\Delta y_{\max }^{\prime}=\frac{e Q \hat{W}_{+} y_{0}}{E}
$$

where $y_{0}$ is the vertical displacement of the electron bunch from the midplane of the small gap chamber. Using the value of $\hat{\mathrm{W}}_{\perp}$ given in Eq. (2.19), we obtain

$$
\Delta y_{\max }^{\prime}=\left(3 \times 10^{-4} \mathrm{rad}\right) \frac{I_{0}(A m p) y_{0}(\mathrm{~mm})}{[a(\mathrm{~mm})]^{3}} .
$$

In the case of $\mathrm{I}_{0}=100 \mathrm{ma}, \mathrm{a}=1 \mathrm{~mm}, \mathrm{y}_{0}=0.1 \mathrm{~mm}$,

$$
\Delta y_{\max }^{\prime}=3 \times 10^{-6} \mathrm{rad}
$$

which is one tenth of the angular spread due to synchrotron radiation. For $\mathrm{I}_{0}=10 \mathrm{ma}, \mathrm{a}=$ $2 \mathrm{~mm}, \mathrm{y}_{0}=0.1 \mathrm{~mm}$,

$$
\Delta y_{\max }^{\prime}=4 \times 10^{-8} \mathrm{rad}
$$

which is very small compared to the angular spread $\sigma_{y}{ }^{\prime}$.

\section{TRANSVERSE GEOMETRIC WAKEFIELD}

Bane and Morton ${ }^{8}$ have considered the transverse wakefield of a scraper, as illustrated in Fig. 2. They considered a perfectly conducting cylindrical tube, and found that when $\sigma_{\mathrm{g}} \geq \mathrm{a}$, the transverse geometric wakefield for a Gaussian bunch is well approximated by

$$
W_{\perp}(s)=\frac{Z_{0} c}{\pi a}\left(\frac{2 \theta}{\pi}\right)^{1 / 2} \frac{1}{\sqrt{2 \pi} \sigma_{s}} e^{-s^{2} / 2 \sigma_{s}^{2}},(b>a) \quad(\theta \text { not too small })
$$

where $Z_{0}=377 \Omega$. Taking $\theta=\pi / 2$ and $\sigma_{s}=0.05 \mathrm{~m}$, we obtain

$$
W_{\perp}(0)=\frac{3 \times 10^{14}}{a(\mathrm{~mm})} \mathrm{Q} / \mathrm{ms}
$$

The result of Eq. (3.2) can be compared with the maximum of the resistive wall wakefield given in Eq. (2.19), $\ell=0.3 \mathrm{~m}$, 


$$
\hat{W}_{\perp} \ell=\frac{1.6 \times 10^{15}}{[a(m m)]^{3}} \Omega / m s
$$

It is seen that for a $=1 \mathrm{~mm}$, the resistive wakefield of Eq. (3.3) is larger than the geometric wakefield of Eq. (3.2). When a $=2 \mathrm{~mm}$, the two are approximately of equal magnitude. For a $>2 \mathrm{~mm}$, the geometric wakefield of Eq. (3.3) will dominate. We can reduce the geometric wakefield by tapering the ends of the small gap chamber, i.e. choosing $\theta$ to be small.

\section{LONGITUDINAL GEOMETRIC WAKEFIELD}

When a bunch passes through the small aperture chamber illustrated in Fig. 2, there is a longitudinal effect due to the longitudinal geometric wakefield. This wakefield has a qualitatively different character depending on whether the bunch length is long, $\sigma_{\mathrm{s}} / \mathrm{b}>1$, or short, $\sigma_{\mathrm{s}} / \mathrm{b}<1$. When $\sigma_{\mathrm{s}} / \mathrm{b}$ is small compared to unity, the beam will radiate energy; when $\sigma_{\mathrm{s}} / \mathrm{b}$ is large almost no energy will be radiated and the wakefield will be inductive.

In the case of the X-Ray ring we can restrict our attention to the regime of a long bunch. For a long bunch, it is known that when $(b-a) / a \leq 1$ the inductance is proportional to $(b-a)^{2}$. For the present application we would like to know the inductance when (b-a)/a is large compared to 1 . To determine this, we carried out a study using the computer program TBCI. ${ }^{10}$ We first determined the wakefield of a long bunch, and fit the result to the derivative of the (Gaussian) bunch distribution in order to find the inductance $\mathrm{L}$ according to

$$
W=-L d I / d t \text {. }
$$

The result is that asymptotically (for very iong bunches), the inductance is well-approximated by

$$
L=\frac{Z_{0} a}{2 \pi c}\left(\frac{b-a}{a}\right)^{5 / s}\left(\frac{2 \theta}{\pi}\right) \quad(\theta \leq \pi / 2)
$$

Fig. 3 shows the computer results as a function of $b-a$, when $\theta=\pi / 2, a=1 \mathrm{~cm}$ and $\sigma_{\mathrm{s}}=10$ $\mathrm{cm}$. The solid line represents Eq. (4.2). Fig. 4 displays the $\theta$-dependence that is obtained when $\mathrm{a}=1 \mathrm{~cm}, \mathrm{~b}=5 \mathrm{~cm}$, and $\sigma_{\mathrm{s}}=10 \mathrm{~cm}$. Again, the solid line represents Eq. (4.2).

We have also considered the sensitivity of the result to bunch length. We find that as long as $\sigma_{\mathrm{s}} / \mathrm{b} \geq 1$, the numerical result is well-approximated by multiplying Eq. (4.2) by the factor

$$
\left[1+\frac{1}{4}\left(\frac{\sigma_{s}}{b}\right)^{-2.5}\right]
$$


To apply these results to the small aperture undulator chamber we consider the parameters $a=2 \mathrm{~mm}, b=1 \mathrm{~cm}$ and $\sigma_{\mathrm{s}}=5 \mathrm{~cm}$. In Fig. 5 , we plot the wakefield for $\theta=$ $90^{\circ}$. The dashes give the Gaussian electron bunch shape. Figure 6 gives, for the same geometry, the inductance as a function of taper angle $\theta$. The solid line gives the result of Eq. (4.2). An inductance of $2 \mathrm{nH}$ is insignificant, corresponding to

$$
Z / n=\omega_{0} L=0.02 \Omega \text {. }
$$

\section{REFERENCES}

1. P.M. Stefan, L. Solomon, S. Krinsky and G. Rakowsky, Proc. 1991 IEEE Part. Accel. Conf., San Francisco, CA, May 6-9, 1991, p. 1096.

2. J.D. Jackson, Classical Electrodynamics, Second Edition, John Wiley and Sons, New York, 1975, Chapter 8.

3. B. Zotter and F. Sacherer, CERN 77-13, p.175.

4. P. Morton and P. Wilson, AATF/79/15, SLAC.

5. E.D. Courant and A.M. Sessler, Rev. Sci. Instrum. 37 (1966), p.1579.

6. K. Bane and M. Sands, Part. Accel. 25 (1990), p.73.

7. R.D. Ruth, Proc. US-CERN School on Part. Accel., Capri, Italy, October 20-26, 1988, Lecture Notes in Physics 343, Springer-Verlag, Berlin, 1989, p.247.

8. K. Bane and P. Morton, Proc. 1986 LINAC Conf., SLAC, June 2-6, 1986, SLAC-303, p.490.

9. K. Bane, Proc. Impedance and Bunch Instability Workshop, Argonne National Laboratory, October 31-November 1, 1989, ANL/APS,'TM-5, p.187.

10. T. Weiland, Nucl. Instrum. Meth. 212 (1983), p.13. 


\section{FIGURE CAPTIONS}

Fig. 1. The function $\mathrm{g}(\xi)$ defined in Eq. (2.15) is plotted for $-3 \leq \xi \leq 3$.

Fig. 2. Profile of cylindrical scraper with inner radius $a$, outer radius $b$, and taper angle $\theta$.

Fig. 3. Inductance $L$ determined from TBCI plotted as function of $b-a$, for $\theta=\pi / 2$, $\mathrm{a}=1 \mathrm{~cm}$ and $\sigma_{\mathrm{s}}=10 \mathrm{~cm}$. Solid line represents Eq. (4.2).

Fig. 4. Inductance $\mathrm{L}$ determined from $\mathrm{TBCI}$ plotted as function of taper angle $\theta$, for $\mathrm{a}=$ $1 \mathrm{~cm}, \mathrm{~b}=5 \mathrm{~cm}$ and $\sigma_{\mathrm{s}}=10 \mathrm{~cm}$. Solid line represents Eq. (4.2).

Fig. 5. Longitudinal wakefield for $\theta=\pi / 2$ determined using TBCI, plotted for PSGU parameters $\mathrm{a}=2 \mathrm{~mm}, \mathrm{~b}=1 \mathrm{~cm}$ and $\sigma_{\mathrm{s}}=5 \mathrm{~cm}$.

Fig. 6. Inductance plotted as function of taper angle $\theta$, for same geometry considered in Fig. 5. Solid line gives the result of Eq. (4.2). 


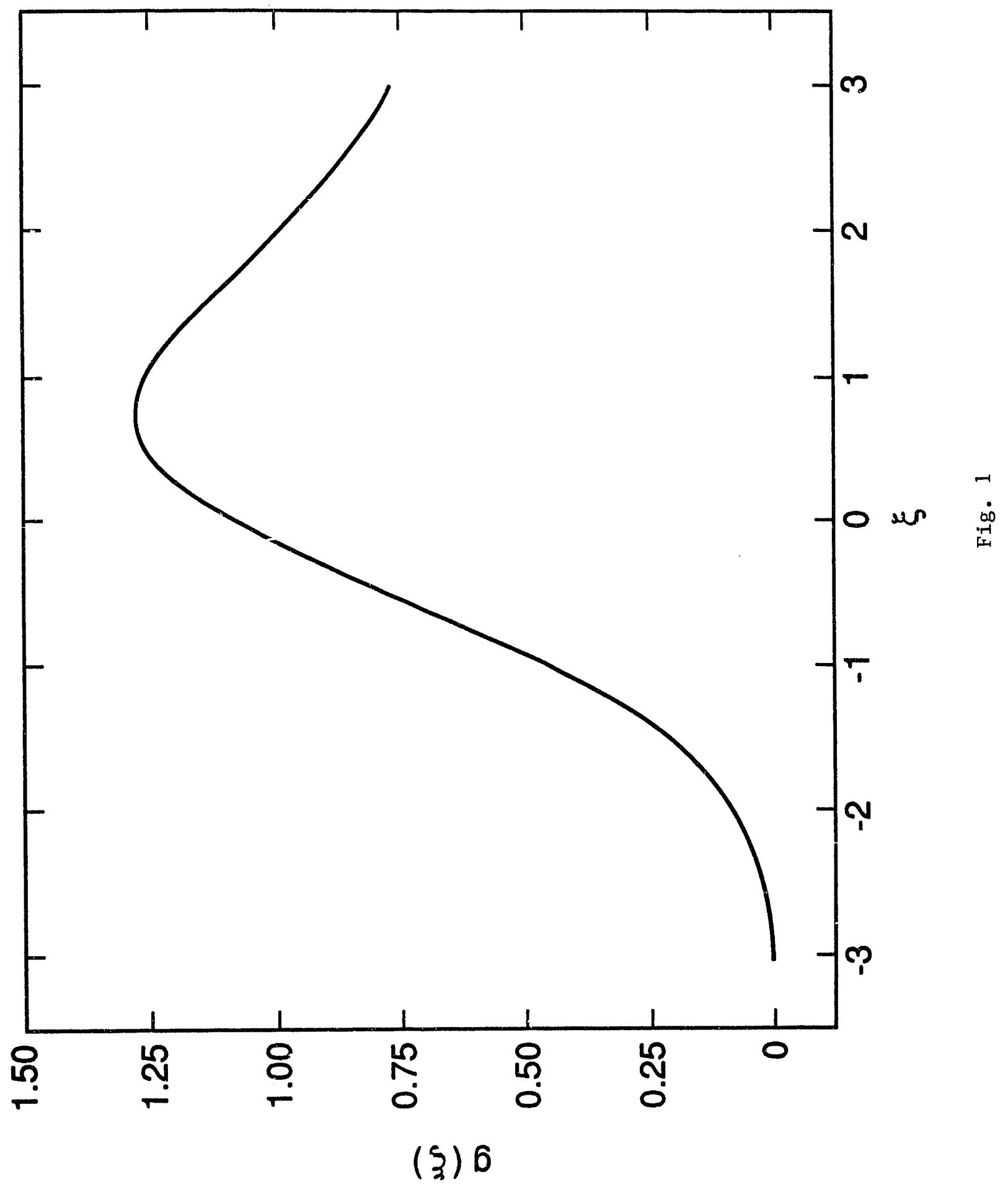




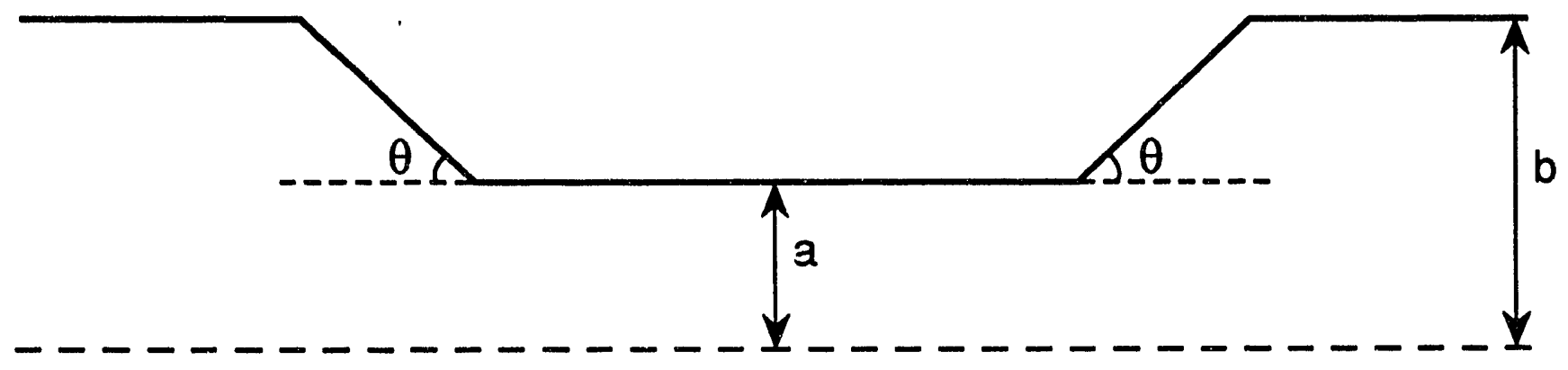

Fig. 2 


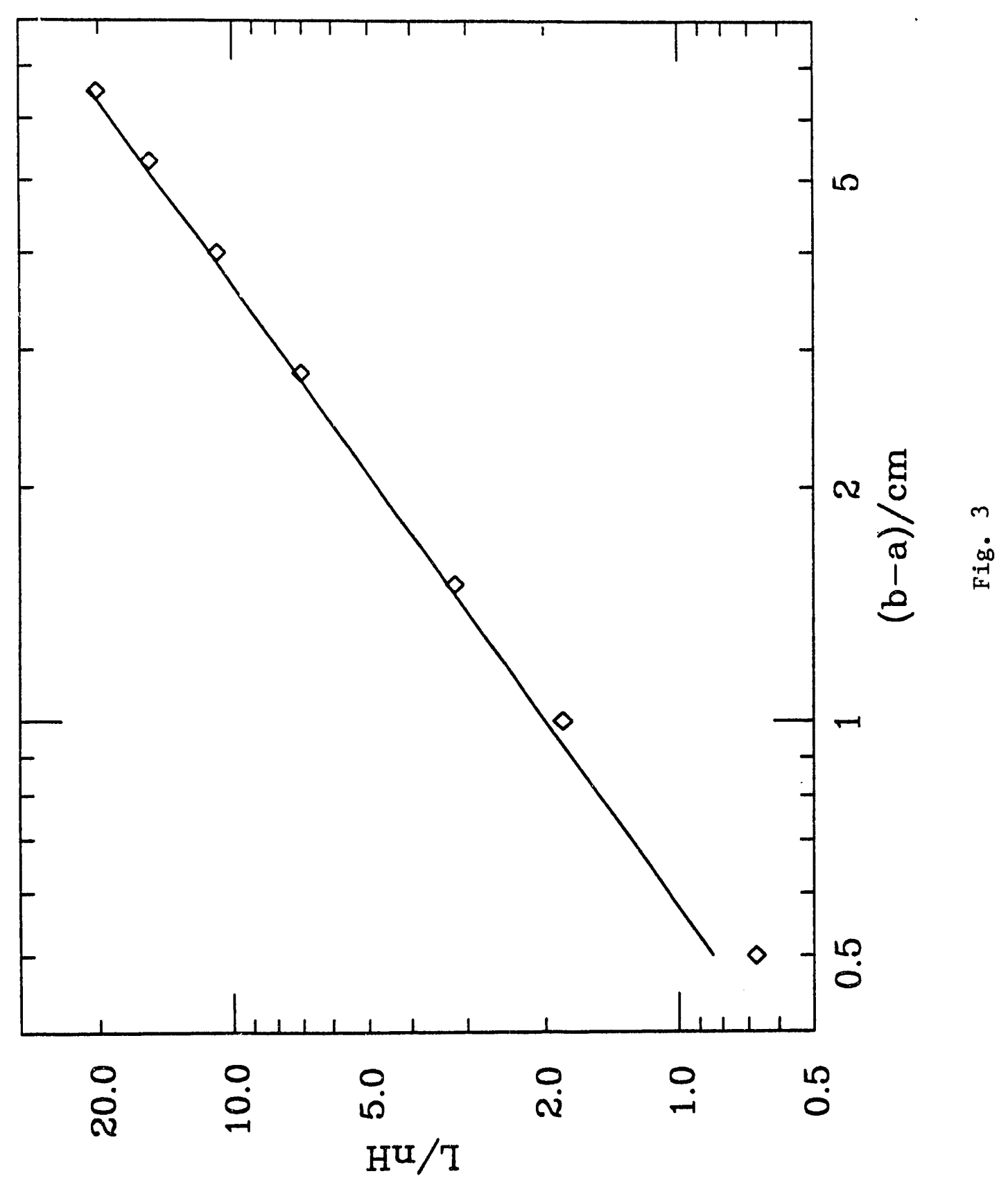




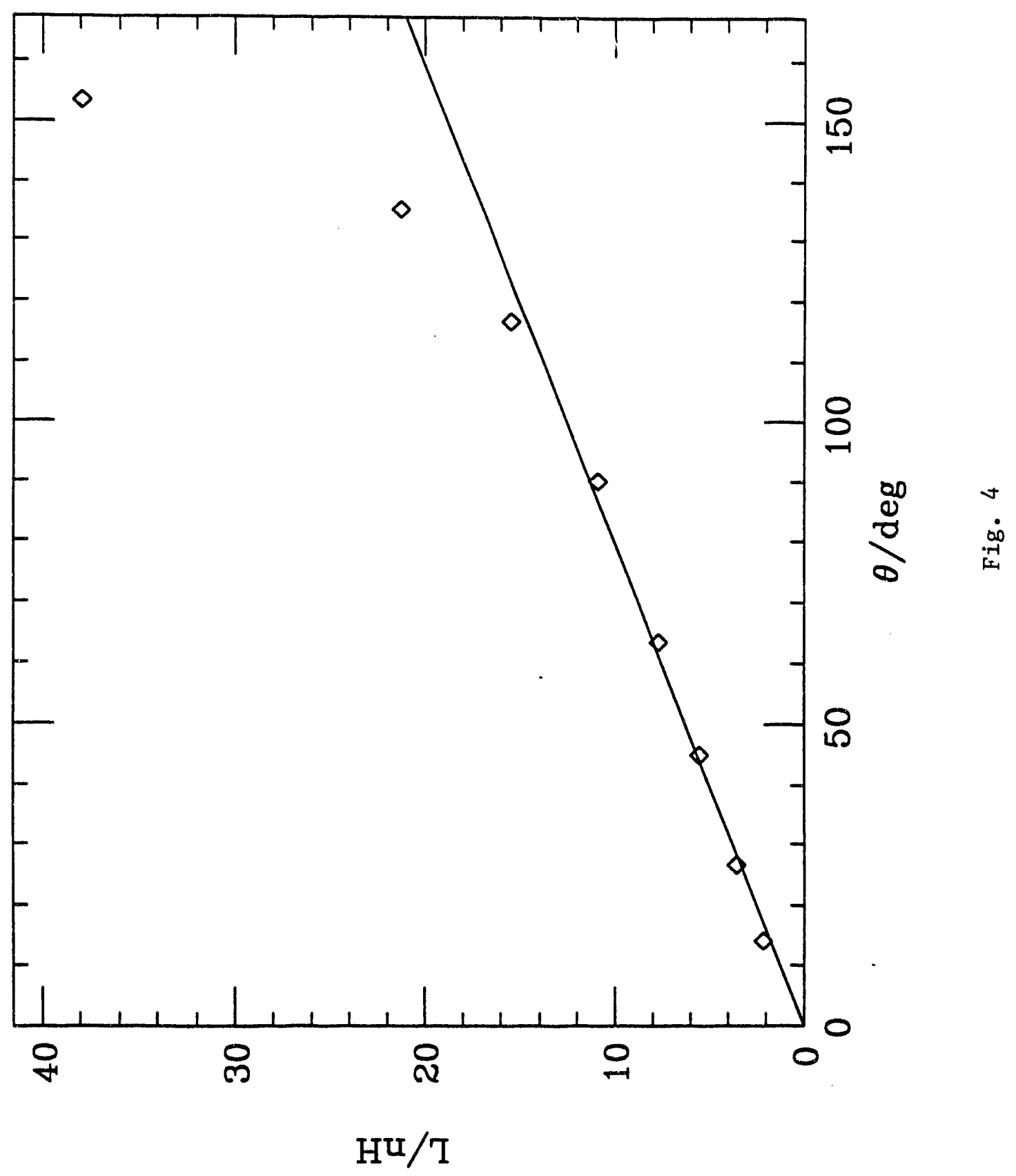




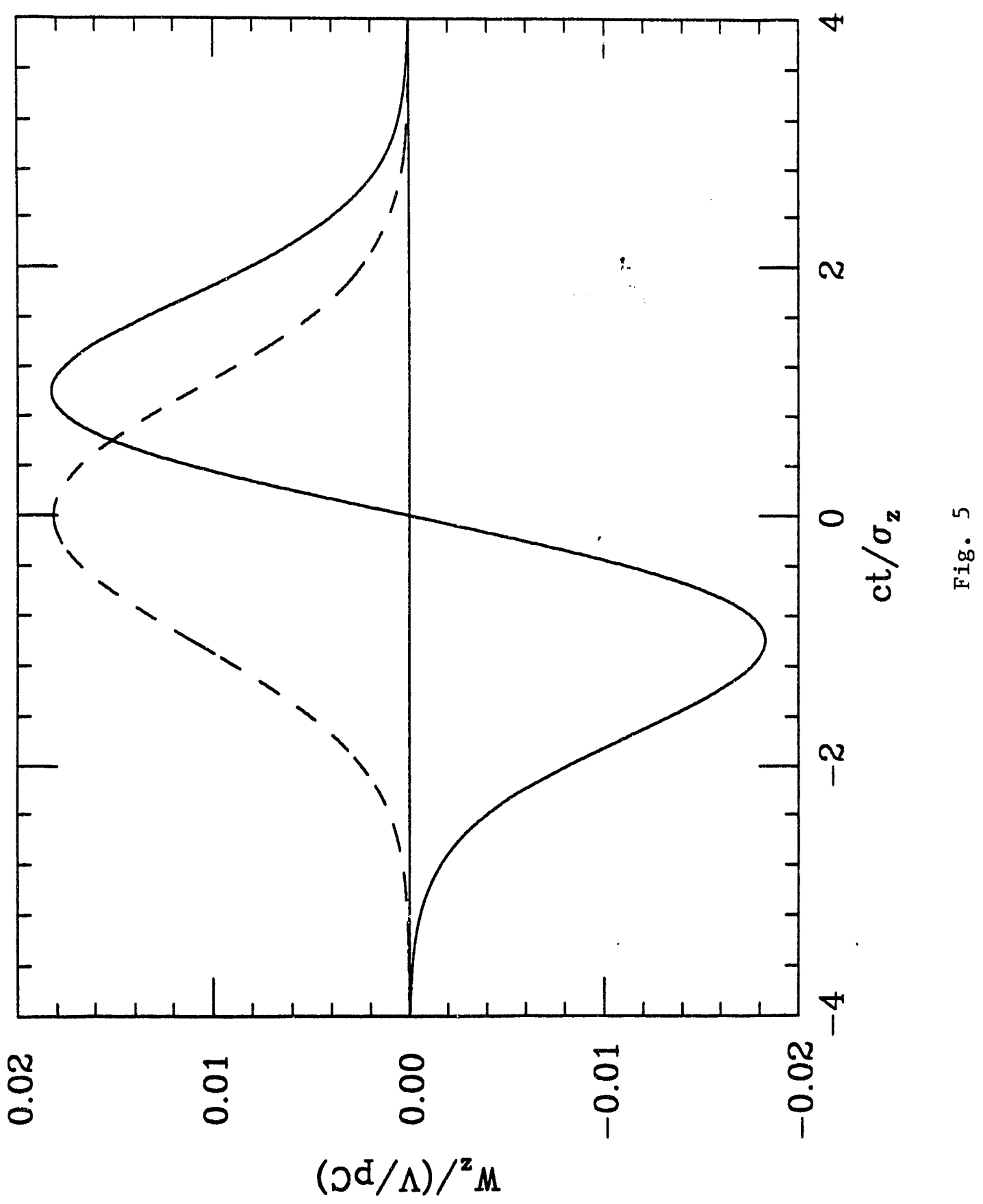




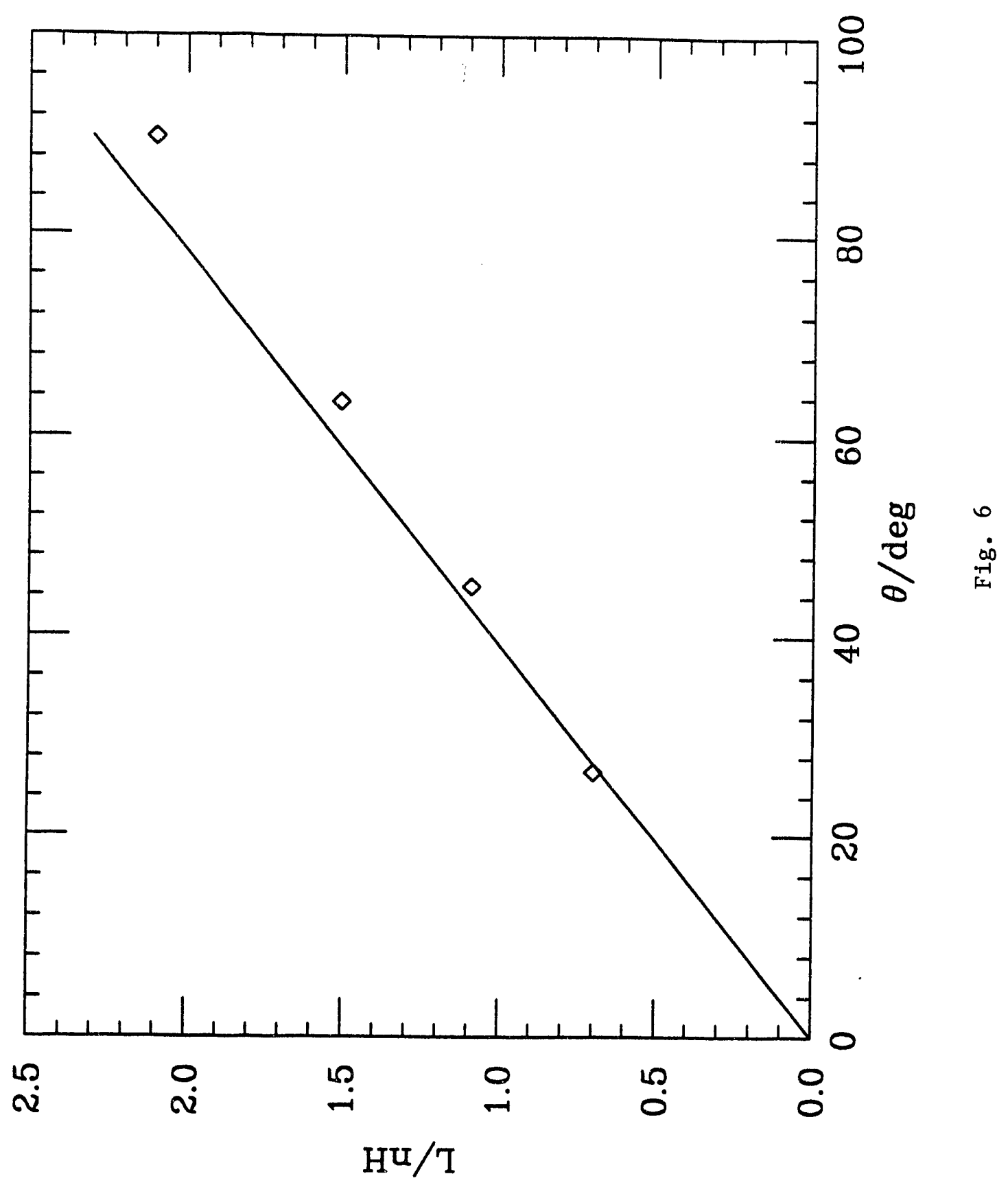



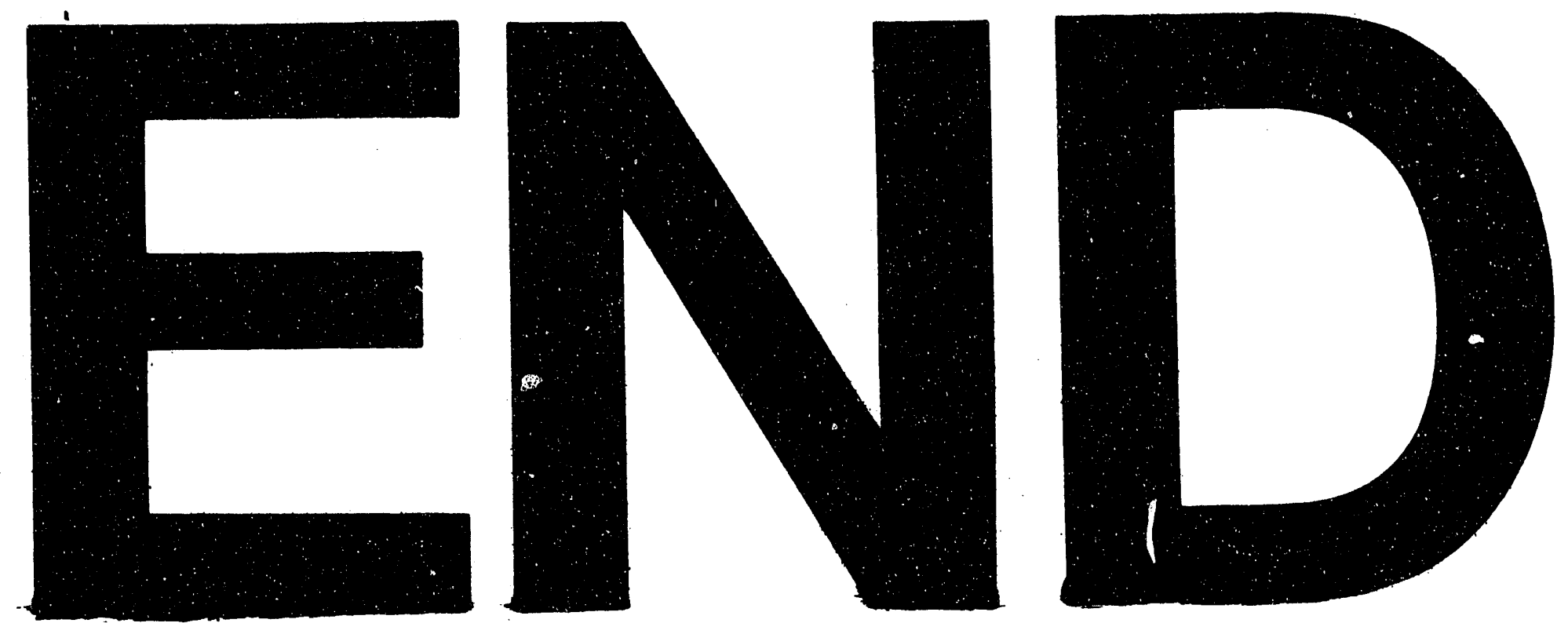

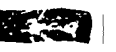

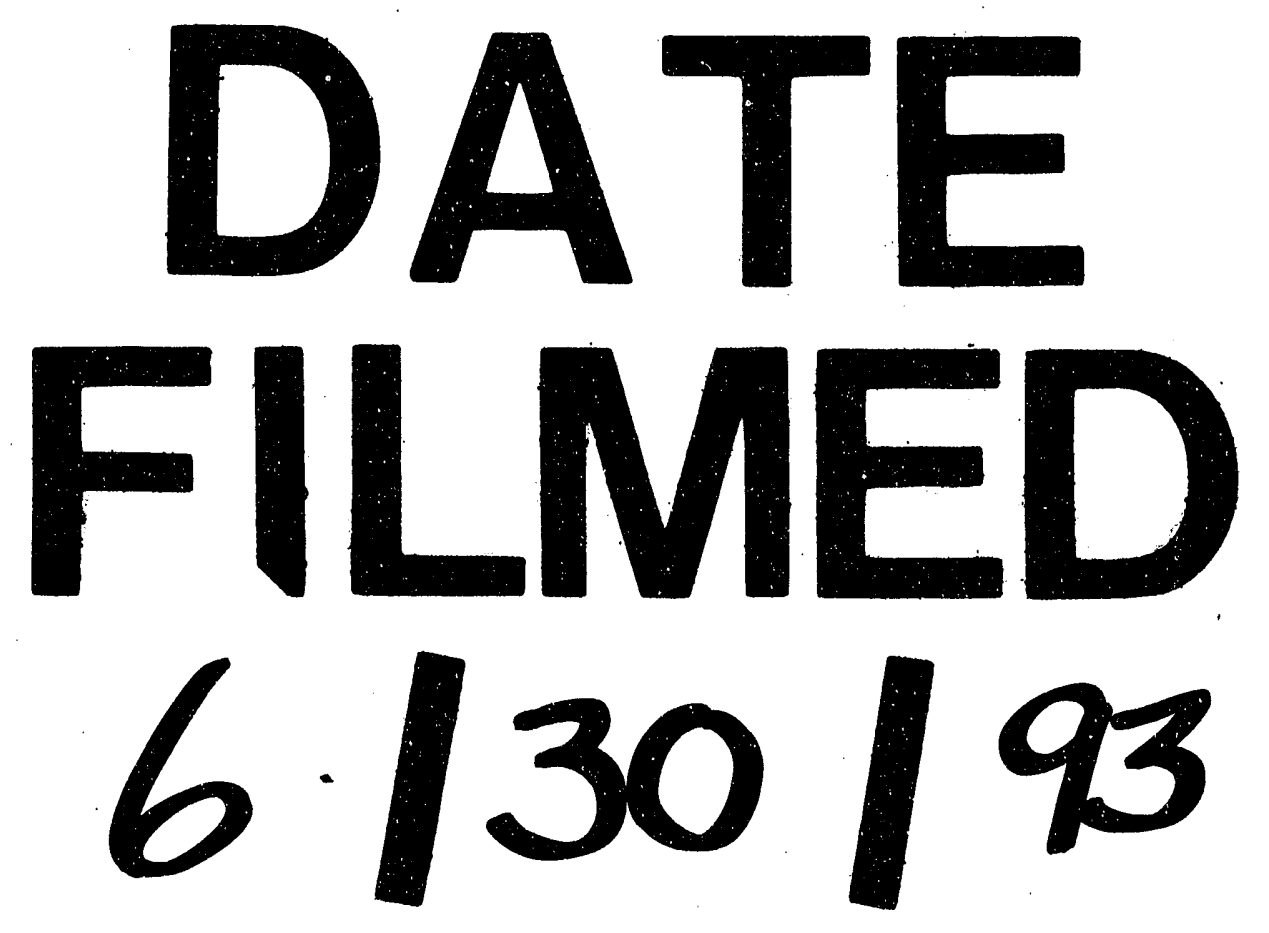


1 